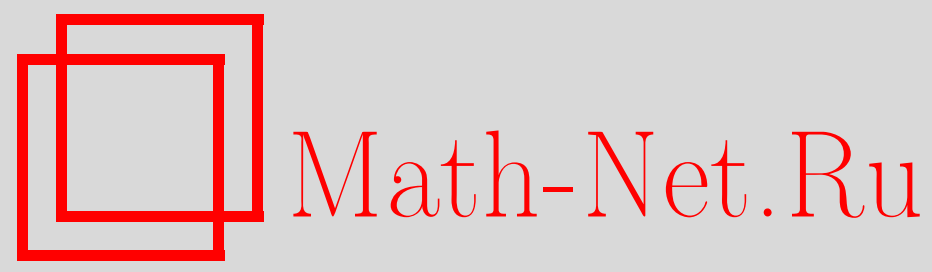

А. А. Саакян, О теореме Бора для кратных рядов Фурье, Матем. заметки, 1998, том 64, выпуск 6, 913-924

DOI: https://doi.org/10.4213/mzm1470

Использование Общероссийского математического портала Math-Net.Ru подразумевает, что вы прочитали и согласны с пользовательским соглашением http://www . mathnet.ru/rus/agreement

Параметры загрузки:

IP: 54.198 .67 .100

26 апреля 2023 г., 13:37:56

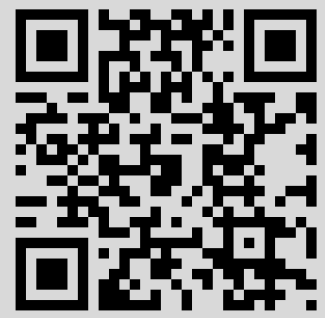




\title{
О ТЕОРЕМЕ БОРА ДЛЯ КРАТНЫХ РЯДОВ ФУРЬЕ
}

\section{А. А. Саакян}

\begin{abstract}
Статья посвящена вопросам равномерной сходимости по Прингсхейму кратных рядов Фурье по тригонометрической системе. Доказан многомерный аналог теоремы Бора о равномерной сходимости ряда Фурье непрерывной функции после гомеоморфной замены переменной.
\end{abstract}

Библиограффия: 7 названий.

1. Введение. Пусть $C(T)\left(C\left(T^{2}\right)\right)$ - пространство непрерывных, $2 \pi$-периодических (по каждой переменной) функций на $\mathbb{R}^{1}\left(\mathbb{R}^{2}\right)$. Для одномерных тригонометрических рядов Фурье хорошо известна (см. [1, с. 303])

Теорема (Г. Бор). Для $f \in C(T)$ существует гомеоморфизм $\tau(t)$ отрезка $T=$ $[-\pi, \pi]$, m.е. непрерывная функиия с условием

$$
-\pi=\tau(-\pi)<\tau\left(t_{1}\right)<\tau\left(t_{2}\right)<\tau(\pi)=\pi, \quad-\pi<t_{1}<t_{2}<\pi,
$$

такая, что ряд Фурье суперпозиции $f$ о $\tau(t)$ равномерно сходится на $T$.

В работах [2], [3] получены разные усиления этой теоремы. В частности, в [2] доказано, что гомеоморфизм $\tau$ в теореме Бора можно построить единым для заданного компакта в $C(T)$.

Теорема (Ж.-П. Кахан, И. Кацнельсон). Пусть

$$
\omega \in C(0, \infty), \quad 0=\omega(0)<\omega\left(\delta_{1}\right)<\omega\left(\delta_{2}\right)<\infty, \quad 0<\delta_{1}<\delta_{2}<\infty .
$$

Существует гомеоморфизм $\tau$ отрезка $T$ такой, что для произвольной функиии $f \in C(T)$ с модулем непрерывности $\omega(\delta, f) \leqslant \omega(\delta)$ ряд Фурье суперпозичии $f \circ \tau(t)$ равномерно сходится на $T$.

В [4] приведен аналог теоремы Бора для кратных тригонометрических рядов Фурье. Однако, как выяснилось, доказательство этого результата содержит пробел: построенная в теореме 2 работы [4] функция $\tau(x)$ не является гомеоморфизмом (условие б) на с. 99 в общем случае не вьполняется; автор благодарит профессора С. В. Конягина, указавшего на эту ошибку).

Сформулированная ниже теорема 1 является обобщением теоремы Кахана и Кацнельсона на многомерный случай и устанавливает, в частности, справедливость теоремы Бора для кратных рядов Фурье. Для $F \in C\left(T^{2}\right)$ обозначим

$$
\omega(\delta, F)=\sup _{\left(x_{1}-x_{2}\right)^{2}+\left(y_{1}-y_{2}\right)^{2} \leqslant \delta^{2}}\left|F\left(x_{1}, y_{1}\right)-F\left(x_{2}, y_{2}\right)\right|, \quad 0<\delta<\infty,
$$


и пусть $S_{n, m}(F, x, y)$ - прямоугольная частная сумма ряда $\Phi$ урье функции $F$ :

$$
S_{n, m}(F, x, y)=\sum_{k=-n}^{n} \sum_{j=-m}^{m} c_{k, j}(F) e^{i(k x+j y)}, \quad(x, y) \in T^{2}, \quad n, m=1,2, \ldots
$$

Напомним, что сходимость ряда Фурье по Прингсхейму означает сходимость прямоугольных частньх сумм при $n, m \rightarrow \infty$.

ТЕорема 1. Для произвольной функиии $\omega(\delta)$ с условиями (2) существует гомеоморфизм $\tau(t)$ отрезка $T$ такой, что ряд Фурье произвольной функиии $F$ вида

$$
F(x, y)=f(\tau(x), \tau(y)), \quad f \in C\left(T^{2}\right), \quad \omega(\delta, f) \leqslant \omega(\delta)
$$

равномерно сходится по Прингсхейму на квадрате $T^{2}$. Более того, для произвольного $\varepsilon>0$ существует номер $N$ такой, что

$$
\left\|S_{n, m}(F)-F\right\|_{C} \leqslant \varepsilon \quad n p u \quad n, m>N
$$

для всех функиий $F$ вида (3).

Для формулировки следующей теоремы нам потребуется ряд обозначений. Через $M(T)\left(M\left(T^{2}\right)\right)$ обозначим пространство $2 \pi$-периодических (по каждой переменной), измеримых, ограниченных на $\mathbb{R}^{1}\left(\mathbb{R}^{2}\right)$ функций $f(x)$, полагая, что

$$
\|f\|_{\infty}=\sup _{x \in \mathbb{R}\left(x \in \mathbb{R}^{2}\right)}|f(x)|
$$

Рассмотрим интервалы

$$
\Delta_{k}^{\delta}(x, n):=\delta\left(x+\frac{2 k-1}{n} \pi, x+\frac{2 k}{n} \pi\right), \quad x \in T, \quad k, n=1,2, \ldots, \quad \delta= \pm 1
$$

где $\delta(a, b)$ - интервал с концами $\delta a$ и $\delta b$.

Для функции $f \in M(T)$ обозначим $f(\Delta)=f(b)-f(a)$, если $\Delta=(a, b)$, и положим

$$
W_{n}^{\delta}(f, x):=\left|\sum_{k=1}^{[n / 2]} \frac{f\left(\Delta_{k}^{\delta}(x, n)\right)}{k}\right|, \quad x \in T, \quad n=1,2, \ldots, \quad \delta= \pm 1
$$

Пусть

$$
W_{n}(f):=\sup _{x \in T, \delta= \pm 1} W_{n}^{\delta}(f, x), \quad n=1,2, \ldots
$$

Для равномерной сходимости одномерных рядов Фурье хорошо известен признак Салема (см. $[1$, с. 283]). 
Teоpema (Р. Салем). Ecлu $f \in C(T) u$

$$
\lim _{n \rightarrow \infty} W_{n}(f)=0
$$

то ряд Фурье функиии $f$ равномерно сходится на T.

Пусть теперь $F(x, y) \in M\left(T^{2}\right)$ и

$$
F\left(\Delta_{1}, \Delta_{2}\right):=F\left(x_{1}, y_{1}\right)+F\left(x_{2}, y_{2}\right)-F\left(x_{1}, y_{2}\right)-F\left(x_{2}, y_{1}\right),
$$

если $\Delta_{1}=\left(x_{1}, y_{1}\right), \Delta_{2}=\left(x_{2}, y_{2}\right)$. Положим

$$
\begin{gathered}
W_{n, m}^{\delta_{1}, \delta_{2}}(F, x, y):=\left|\sum_{k=1}^{[n / 2]} \sum_{j=1}^{[m / 2]} \frac{F\left(\Delta_{k}^{\delta_{1}}(x, n), \Delta_{j}^{\delta_{2}}(y, m)\right)}{k j}\right|, \\
(x, y) \in T^{2}, \quad n, m=1,2, \ldots, \quad \delta_{1}, \delta_{2}= \pm 1 .
\end{gathered}
$$

Пусть

$$
W_{n, m}(F):=\sup _{(x, y) \in T^{2}, \delta_{1}, \delta_{2}= \pm 1}\left\{W_{n, m}^{\delta_{1}, \delta_{2}}(F, x, y)+W_{n}^{\delta_{1}}(F(\cdot, y), x)+W_{m}^{\delta_{2}}(F(x, \cdot), y)\right\} .
$$

Следующая теорема является аналогом признака Салема для двойных рядов Фурье. Она была доказана в работе [5], но мы приводим ее с полным доказательством, так как при обосновании второго утверждения теоремы 1 (см. (4)), кроме равномерной сходимости ряда $\Phi$ урье, нам понадобится оценка, полученная в лемме 4.

Теорема 2 (Б.И. Голубов). $E c л u F(x, y) \in C\left(T^{2}\right) u$

$$
\lim _{n, m \rightarrow \infty} W_{n, m}(F)=0
$$

то ряд Фурье функиии F равномерно сходится по Прингсхейму на $T^{2}$.

ЗАмЕчАниЕ 1. Для краткости изложения мы приводим теоремы 1 и 2 в двумерном случае, но их аналоги справедливы в $\mathbb{R}^{k}$ при любом $k \geqslant 2$ и доказываются аналогично. Более того, функция $\tau$ в теореме 1 может быть построена не зависящей от размерности пространства.

2. Вспомогательные утверждения. Всюду ниже через $C$ мы обозначаем абсолютные константы, которые могут быть разными даже в одной и той же формуле. Выражение вида $O(\Omega)$ означает величину, удовлетворяющую условию

$$
|O(\Omega)| \leqslant C|\Omega| .
$$

Доказательство следующей леммы фактически содержится в доказательстве признака Салема (см. [1, с. 284-286]), но мы приводим его для полноты изложения.

Лемма 1. Существует убьвающая последовательность $\left\{\eta_{n}\right\}$ с $\lim _{n \rightarrow \infty} \eta_{n}=0$ такая, что для произвольной функиии $\varphi \in M(T)$ справедливо соотношение

$$
\frac{1}{\pi} \int_{-\pi}^{\pi} \varphi(x+t) \frac{\sin n t}{t} d t=\varphi(x)+O\left(U_{n}(\varphi)\right), \quad x \in T, \quad n=1,2, \ldots,
$$

əде

$$
U_{n}(\varphi):=W_{n}(\varphi)+\omega\left(\frac{\ln n}{n}, \varphi\right)+\|\varphi\|_{\infty} \eta_{n}
$$


ДокаЗАТЕЛЬСТво. Пусть $x \in T$ и $n=2 n_{0}+1+\gamma, \gamma \in\{0,1\}$, фиксированы. Положим

$$
\psi(t):=\varphi(x+t)-\varphi(x), \quad \eta_{n}^{\prime}:=\frac{1}{\pi} \int_{-\pi}^{\pi} \frac{\sin n t}{t} d t-1 .
$$

Тогда $|\psi(t)| \leqslant \omega(|t|, \varphi), t \in T, \lim _{n \rightarrow \infty} \eta_{n}^{\prime}=0$ и

$$
\frac{1}{\pi} \int_{-\pi}^{\pi} \varphi(x+t) \frac{\sin n t}{t} d t-\varphi(x)=\frac{1}{\pi} \int_{-\pi}^{\pi} \psi(t) \frac{\sin n t}{t} d t+\eta_{n}^{\prime} \varphi_{n}(x) .
$$

Оценим интеграл по $[0, \pi]$ (интеграл по $[-\pi, 0]$ оценивается аналогично):

$$
\begin{aligned}
& \left|\int_{0}^{\pi} \psi(t) \frac{\sin n t}{t} d t\right| \leqslant\left|\sum_{k=0}^{2 n_{0}+\gamma} \int_{k \pi / n}^{(k+1) \pi / n} \psi(t) \frac{\sin n t}{t} d t\right| \\
& \quad \leqslant \pi\|\psi\|_{L^{\infty}(0, \pi / n)}+\frac{\pi}{n}\|\psi\|_{\infty}+\left|\sum_{k=1}^{2 n_{0}} \int_{0}^{\pi} \psi\left(\frac{t+k \pi}{n}\right) \frac{\sin (t+k \pi)}{t+k \pi} d t\right| \\
& \quad \leqslant \omega\left(\frac{\pi}{n}, \varphi\right)+\frac{2 \pi}{n}\|\varphi\|_{\infty}+\int_{0}^{\pi}\left|\sum_{k=1}^{n_{0}}\left(\frac{\psi((t+2 k \pi) / n)}{t+2 k \pi}-\frac{\psi((t+(2 k-1) \pi) / n)}{t+(2 k-1) \pi}\right)\right| d t \\
& \quad \leqslant \omega\left(\frac{\pi}{n}, \varphi\right)+\frac{2 \pi}{n}\|\varphi\|_{\infty}+\pi \sup _{t \in[0, \pi]}\left|\sum_{k=1}^{n_{0}} I_{k}(t)\right|
\end{aligned}
$$

где

$$
I_{k}(t):=\frac{\psi((t+2 k \pi) / n)}{t+2 k \pi}-\frac{\psi((t+(2 k-1) \pi) / n)}{t+(2 k-1) \pi} .
$$

Представим величину $I_{k}(t)$ в виде

$$
\begin{aligned}
I_{k}(t)= & \frac{\psi((t+2 k \pi) / n)-\psi((t+(2 k-1) \pi) / n)}{t+2 k \pi}-\frac{\pi \psi((t+(2 k-1) \pi) / n)}{(t+2 k \pi)(t+(2 k-1) \pi)} \\
= & \frac{1}{2 \pi} \frac{\varphi((t+2 k \pi) / n)-\varphi((t+(2 k-1) \pi) / n)}{k} \\
& -\frac{t}{2 \pi} \frac{\varphi((t+2 k \pi) / n)-\varphi((t+(2 k-1) \pi) / n)}{k(t+2 k \pi)}-\frac{\pi \psi((t+(2 k-1) \pi) / n)}{(t+2 k \pi)(t+(2 k-1) \pi)} \\
= & \frac{1}{2 \pi} \frac{\varphi((t+2 k \pi) / n)-\varphi((t+(2 k-1) \pi) / n)}{k}+I_{k}^{\prime}(t)+I_{k}^{\prime \prime}(t) .
\end{aligned}
$$

Далее, имеем

$$
\left|\sum_{k=1}^{n_{0}} I_{k}^{\prime}(t)\right| \leqslant C \sum_{k=1}^{\infty} \frac{\omega(\pi / n, \varphi)}{k^{2}} \leqslant C \omega\left(\frac{1}{n}, \varphi\right)
$$

и, наконец,

$$
\begin{aligned}
\left|\sum_{k=1}^{n_{0}} I_{k}^{\prime \prime}(t)\right| & \leqslant C\left(\sum_{k=1}^{[\ln n]} \frac{\omega((\pi \ln n) / n, \varphi)}{k^{2}}+\|\varphi\|_{\infty} \sum_{k=[\ln n]+1}^{\infty} \frac{1}{k^{2}}\right) \\
& \leqslant C\left(\omega\left(\frac{\ln n}{n}, \varphi\right)+\|\varphi\|_{\infty} \eta_{n}^{\prime \prime}\right)
\end{aligned}
$$


где

$$
\eta_{n}^{\prime \prime}:=\sum_{k=[\ln n]+1}^{\infty} \frac{1}{k^{2}} \rightarrow 0 \quad \text { при } n \rightarrow \infty .
$$

Из (7)-(11) получим (6) с $\eta_{n}=\max _{k \geqslant n}\left\{\eta_{k}^{\prime}+\eta_{k}^{\prime \prime}\right\}$. Лемма 1 доказана.

Обозначим через $\omega_{1}(\delta, f)$ интегральньй модуль непрерьвности функции $f$. Для коэффициентов Фурье хорошо известна оценка (см. $[1$, с. 80])

$$
\left|c_{m}(u)\right| \leqslant \omega_{1}\left(\frac{1}{m}, u\right) .
$$

Обозначим также

$$
g(t):=\frac{1}{2 \operatorname{tg}(t / 2)}-\frac{1}{t}, \quad 0<|t| \leqslant \pi, \quad g(0)=0 .
$$

Лемма 2. Существует постоянная $K>0$ такая, что для произвольной функиии $F \in M\left(T^{2}\right)$ имеет место неравенство

$$
\begin{gathered}
\left|\int_{-\pi}^{\pi} \int_{-\pi}^{\pi} F(x+t, y+s) g(s) \frac{\sin n t}{t} e^{i m s} d t d s\right| \leqslant K U_{n, m}(F), \\
(x, y) \in T^{2}, \quad n, m=1,2, \ldots
\end{gathered}
$$

$2 \partial e$

$$
U_{n, m}(F):=W_{n, m}(F)+\omega\left(\frac{\ln n}{n}+\frac{\ln m}{m}, F\right)+\|F\|_{\infty} \gamma_{n, m}
$$

$u($ см. лемму 1$)$

$$
\gamma_{n, m}:=\omega_{1}\left(\frac{1}{n}+\frac{1}{m}, g\right)+\eta_{n}+\eta_{m} \rightarrow 0 \quad \text { npu } \quad n, m \rightarrow \infty .
$$

ДокАЗАТЕЛЬСТво. Согласно лемме 1

$$
\int_{-\pi}^{\pi} F(x+t, y+s) \frac{\sin n t}{t} d t=F(x, y+s)+O\left(U_{n, m}(F)\right) .
$$

Значит, необходимо оценить коэффициенты eM

$$
\begin{aligned}
|u(s+h)-u(s)| \leqslant & |F(x, y+s+h)-F(x, y+s)| \cdot|g(s+h)| \\
& +|F(x, y+s)| \cdot|g(s+h)-g(s)| \\
\leqslant & \|g\|_{\infty} \omega(h, F)+\|F\|_{\infty}|g(s+h)-g(s)| .
\end{aligned}
$$

Тем самьм,

$$
\omega_{1}(\delta, u) \leqslant C\left(\omega(\delta, F)+\|F\|_{\infty} \omega_{1}(\delta, g)\right), \quad \delta>0 .
$$

Отсюда с учетом оценки (12) вытекает (14). Лемма 2 доказана.

ЗАмЕчАниЕ 2. Легко видеть, что оценка (14) остается в силе, если вместо функции $g(t)$, определенной в $(13)$, взять $g(t) \equiv 1$. 
3. Доказательство теорем. Утверждение теоремы 2 непосредственно вытекает из следующей ниже леммы 4 . Сначала мы докажем, что справедлива

ЛЕмма 3. Для произвольных $F \in M\left(T^{2}\right),(x, y) \in T^{2}, n, m=1,2, \ldots$, имеет место соотношение

$$
S_{n, m}(F, x, y)=\frac{1}{\pi^{2}} \int_{-\pi}^{\pi} \int_{-\pi}^{\pi} F(x+t, y+s) \frac{\sin n t}{t} \frac{\sin m s}{s} d t d s+O\left(U_{n, m}(F)\right),
$$

где $U_{n, m}(F)$ определяется формулой (15).

ДокАЗАТЕЛЬСтво. Имеем равенство

$$
S_{n, m}(F, x, y)=\frac{1}{\pi^{2}} \int_{-\pi}^{\pi} \int_{-\pi}^{\pi} F(x+t, y+s) D_{n}(t) D_{m}(s) d t d s,
$$

где $D_{n}$ - ядро Дирихле:

$$
D_{n}(t)=\frac{\sin (n+1 / 2) t}{2 \sin (t / 2)}, \quad t \in T \text {. }
$$

Применив равенство (см. (13))

$$
D_{n}(t)=\frac{\sin n t}{t}+g(t) \sin n t+\frac{1}{2} \cos n t, \quad t \in T,
$$

из (18) получим

$$
\begin{aligned}
\pi^{2} S_{n, m}(F, x, y) & =\int_{-\pi}^{\pi} \int_{-\pi}^{\pi} F(x+t, y+s) \frac{\sin n t}{t} \frac{\sin m s}{s} d t d s \\
& +\int_{-\pi}^{\pi} \int_{-\pi}^{\pi} F(x+t, y+s) \frac{\sin n t}{t}\left(g(s) \sin m s+\frac{1}{2} \cos m s\right) d t d s \\
& +\int_{-\pi}^{\pi} \int_{-\pi}^{\pi} F(x+t, y+s) \frac{\sin m s}{s}\left(g(t) \sin n t+\frac{1}{2} \cos n t\right) d t d s \\
& +\int_{-\pi}^{\pi} \int_{-\pi}^{\pi} F(x+t, y+s)\left(g(t) \sin n t+\frac{1}{2} \cos n t\right)\left(g(s) \sin m s+\frac{1}{2} \cos m s\right) d t d s \\
= & \int_{-\pi}^{\pi} \int_{-\pi}^{\pi} F(x+t, y+s) \frac{\sin n t}{t} \frac{\sin m s}{s} d t d s+\sum_{p=1}^{3} Q_{n, m}^{(p)}(x, y) .
\end{aligned}
$$

Остается доказать, что при $p=1,2,3$

$$
Q_{n, m}^{(p)}(x, y)=O\left(U_{n, m}(F)\right), \quad(x, y) \in T^{2}, \quad n, m=1,2, \ldots
$$

При $p=1$ и $p=2$ это вытекает из леммы 2 (см. также замечание 2 ), а при $p=3-$ из (12) и (16). Лемма 3 доказана.

Лемма 4. Для произвольной $F \in M\left(T^{2}\right)$ имеет место соотношение

$$
S_{n, m}(F, x, y)=F(x, y)+O\left(U_{n, m}(F)\right), \quad(x, y) \in T^{2}, \quad n, m=1,2, \ldots,
$$

где $U_{n, m}(F)$ определяется формулой $(15)$. 
ДокАЗАТЕЛЬСтво. Согласно лемме 3 мы докажем лемму 4, если покажем, что

$$
\frac{1}{\pi^{2}} \int_{-\pi}^{\pi} \int_{-\pi}^{\pi} F(x+t, y+s) \frac{\sin n t}{t} \frac{\sin m s}{s} d t d s=F(x, y)+O\left(U_{n, m}(F)\right) .
$$

Соотношение (19) достаточно получить в случае интеграла по прямоугольнику $[0, \pi] \times$ $[0, \pi]$, т.е. доказать, что

$$
J:=\frac{1}{\pi^{2}} \int_{0}^{\pi} \int_{0}^{\pi} F(x+t, y+s) \frac{\sin n t}{t} \frac{\sin m s}{s} d t d s-\frac{1}{4} F(x, y)=O\left(U_{n, m}(F)\right) .
$$

Обозначим $\Phi(t, s):=F(x+t, y+s)-F(x, y)$. Пусть $n=2 n_{0}+1+\gamma, m=2 m_{0}+1+\sigma$, $\gamma, \sigma \in\{0,1\}$. Согласно (17) (c $F \equiv 1)$ имеем

$$
\begin{aligned}
\pi^{2} J= & \int_{0}^{\pi} \int_{0}^{\pi} \Phi(t, s) \frac{\sin n t}{t} \frac{\sin m s}{s} d t d s+O\left(U_{n, m}(F)\right) \\
= & \int_{0}^{\pi / m} \int_{0}^{\pi / n}+\int_{0}^{\pi / m} \int_{\pi / n}^{\pi}+\int_{\pi / m}^{\pi} \int_{0}^{\pi / n}+\int_{\left(2 m_{0}+1\right) \pi / m}^{\pi} \int_{\pi / n}^{\pi}+\int_{\pi / n}^{\left(2 m_{0}+1\right) \pi / m}+O\left(U_{n, m}(F)\right) \\
& \left.+\int_{\pi / m}^{\left(2 m_{0}+1\right) \pi / n}+1\right) \pi / m \\
= & : \sum_{p=1}^{6} J_{p}+O\left(U_{n, m}(F)\right) .
\end{aligned}
$$

Докажем, что

$$
\left|J_{p}\right|=O\left(U_{n, m}(F)\right), \quad p=1, \ldots, 6 .
$$

При $p=1$ условие (20) очевидно. Согласно (6) для любого $s \in T$

$$
\int_{\pi / n}^{\pi} \Phi(t, s) \frac{\sin n t}{t} d t=O\left(U_{n}(F(x+\cdot, y+s), t)\right)
$$

откуда вытекает (20) при $p=2$ и $p=4$. Аналогично доказывается (20) при $p=3$ и $p=5$. Осталось рассмотреть случай $p=6$. Имеем

$$
\begin{aligned}
J_{6} & =\sum_{k=1}^{2 n_{0}} \sum_{j=1}^{2 m_{0}} \int_{j \pi / m}^{(j+1) \pi / m} \int_{k \pi / n}^{(k+1) \pi / n} \Phi(t, s) \frac{\sin n t}{t} \frac{\sin m s}{s} d t d s \\
& =\int_{0}^{\pi} \int_{0}^{\pi} \sum_{k=1}^{n_{0}} \sum_{j=1}^{m_{0}} I_{k, j}(t, s) \sin t \sin s d t d s
\end{aligned}
$$

где

$$
\begin{aligned}
& I_{k, j}(t, s):=\frac{\Phi((t+2 k \pi) / n,(s+2 j \pi) / m)}{(t+2 k \pi)(s+2 j \pi)}-\frac{\Phi((t+(2 k-1) \pi) / n,(s+2 j \pi) / m)}{(t+(2 k-1) \pi)(s+2 j \pi)} \\
& \quad-\frac{\Phi((t+2 k \pi) / n,(s+(2 j-1) \pi) / m)}{(t+2 k \pi)(s+(2 j-1) \pi)}+\frac{\Phi((t+(2 k-1) \pi) / n,(s+(2 j-1) \pi) / m)}{(t+(2 k-1) \pi)(s+(2 j-1) \pi)} .
\end{aligned}
$$

Обозначим

$$
\Delta_{h}^{(1)} \Phi(t, s)=\Phi(t+h, s)-\Phi(t, s), \quad \Delta_{h}^{(2)} \Phi(t, s)=\Phi(t, s+h)-\Phi(t, s) .
$$


Ясно, что

$$
\left|\Delta_{h}^{(1)} \Phi(t, s)\right|=\left|\Delta_{h}^{(1)} F(t, s)\right| \leqslant \omega(h, F), \quad|\Phi(t, s)| \leqslant \omega\left(\left(t^{2}+s^{2}\right)^{1 / 2}, F\right) .
$$

Величину $I_{k, j}(t, s)$ можно представить в виде

$$
\begin{aligned}
I_{k, j}(t, s)= & \frac{1}{(t+2 k \pi)(s+2 j \pi)} \Delta_{\pi / n}^{(1)} \Delta_{\pi / m}^{(2)} \Phi\left(\frac{t+2 k \pi}{n}, \frac{s+2 j \pi}{m}\right) \\
& +\frac{\pi}{(t+2 k \pi)(s+2 j \pi)(s+(2 j-1) \pi)} \Delta_{\pi / n}^{(1)} \Phi\left(\frac{t+2 k \pi}{n}, \frac{s+(2 j-1) \pi}{m}\right) \\
& +\frac{\pi}{(t+2 k \pi)(t+(2 k-1) \pi)(s+2 j \pi)} \Delta_{\pi / m}^{(2)} \Phi\left(\frac{t+(2 k-1) \pi}{n}, \frac{s+2 j \pi}{m}\right) \\
& +\frac{\pi^{2}}{(t+2 k \pi)(t+(2 k-1) \pi)(s+2 j \pi)(s+(2 j-1) \pi)} \Phi\left(\frac{t+(k+1) \pi}{n}, \frac{s+j \pi}{m}\right) \\
= & : \sum_{p=1}^{4} I_{k, j}^{(p)}(t, s) .
\end{aligned}
$$

Для завершения доказательства леммы 4 нам с учетом (19) и (21) остается показать, что

$$
\sum_{k=1}^{n_{0}} \sum_{j=1}^{m_{0}} I_{k, j}^{(p)}(t, s)=O\left(U_{n, m}(F)\right), \quad p=1,2,3,4
$$

Для доказательства этого соотношения при $p=1$ воспользуемся равенством (здесь $k, j=1,2, \ldots, t, s \in[0, \pi])$

$$
\begin{aligned}
\frac{1}{(t+2 k \pi)(s+2 j \pi)}= & \frac{1}{4 \pi^{2} k j}-\frac{s}{4 \pi^{2} j(s+2 j \pi) k}-\frac{t}{4 \pi^{2} k(s+2 k \pi) j} \\
& +\frac{t s}{4 \pi^{2} j(s+2 j \pi) k(t+2 k \pi)} \\
= & \frac{1}{4 \pi^{2} k j}+O\left(\frac{1}{j^{2}}\right) \frac{1}{k}+O\left(\frac{1}{k^{2}}\right) \frac{1}{j}+O\left(\frac{1}{k^{2} j^{2}}\right) .
\end{aligned}
$$

Следовательно,

$$
\begin{aligned}
\left|\sum_{k=1}^{n_{0}} \sum_{j=1}^{m_{0}} I_{k, j}^{(1)}(t, s)\right| \leqslant & \frac{1}{4 \pi^{2}}\left|\sum_{k=1}^{n_{0}} \sum_{j=1}^{m_{0}} \frac{1}{k j} \Delta_{\pi / n}^{(1)} \Delta_{\pi / m}^{(2)} \Phi\left(\frac{t+2 k \pi}{n}, \frac{s+2 j \pi}{m}\right)\right| \\
& +\left|\sum_{j=1}^{m_{0}} O\left(\frac{1}{j^{2}}\right) \Delta_{\pi / m}^{(2)} \sum_{k=1}^{n_{0}} \frac{1}{j} \Delta_{\pi / n}^{(1)} \Phi\left(\frac{t+2 k \pi}{n}, \frac{s+2 j \pi}{m}\right)\right| \\
& +\left|\sum_{k=1}^{n_{0}} O\left(\frac{1}{k^{2}}\right) \Delta_{\pi / m}^{(1)} \sum_{k=1}^{n_{0}} \frac{1}{j} \Delta_{\pi / n}^{(2)} \Phi\left(\frac{t+2 k \pi}{n}, \frac{s+2 j \pi}{m}\right)\right| \\
& +\left|\sum_{k=1}^{n_{0}} \sum_{j=1}^{m_{0}} O\left(\frac{1}{k^{2} j^{2}}\right) \Delta_{\pi / n}^{(1)} \Delta_{\pi / m}^{(2)} \Phi\left(\frac{t+2 k \pi}{n}, \frac{s+2 j \pi}{m}\right)\right|
\end{aligned}
$$




$$
\begin{aligned}
& \leqslant C W_{n, m}(F)\left(\frac{1}{4 \pi^{2}}+2 \sum_{j=1}^{\infty} \frac{1}{j^{2}}+2 \sum_{k=1}^{\infty} \frac{1}{k^{2}}\right)+C \omega\left(\frac{\pi}{n}+\frac{\pi}{m}, F\right) \sum_{k=1}^{\infty} \sum_{j=1}^{\infty} \frac{1}{k^{2} j^{2}} \\
& \leqslant C U_{n, m}(F) .
\end{aligned}
$$

Соотношение (23) при $p=2$ и $p=3$ доказьвается аналогично с использованием равенств (сравни с (24))

$$
\begin{aligned}
& \frac{1}{(t+2 k \pi)(s+2 j \pi)(s+(2 j-1) \pi)}=O\left(\frac{1}{j^{2}}\right) \frac{1}{k}+O\left(\frac{1}{k^{2} j^{2}}\right) \\
& \frac{1}{(t+2 k \pi)(s+(2 k-1) \pi)(s+2 j \pi)}=O\left(\frac{1}{k^{2}}\right) \frac{1}{j}+O\left(\frac{1}{k^{2} j^{2}}\right) .
\end{aligned}
$$

Остается доказать (23) при $p=4$. С учетом (22) имеем

$$
\begin{aligned}
\left|I_{k, j}^{(4)}(t, s)\right| \leqslant & \|\Phi\|_{C((0,(\pi \ln n) / n) \times(0,(\pi \ln m) / m))} \sum_{k=1}^{[\ln n]} \sum_{j=1}^{[\ln m]} \frac{1}{k^{2} j^{2}} \\
& +2\|\Phi\|_{\infty}\left(\sum_{k=[\ln n]+1}^{\infty} \sum_{j=1}^{\infty} \frac{1}{k^{2} j^{2}}+\sum_{k=1}^{[\ln n]} \sum_{j=[\ln m]+1}^{\infty} \frac{1}{k^{2} j^{2}}\right) \\
\leqslant & C\left(\omega\left(\frac{\pi}{n}+\frac{\pi}{m}, F\right)+\|F\|_{\infty}\left(\eta_{n}+\eta_{m}\right)\right) \leqslant C U_{n, m}(F) .
\end{aligned}
$$

Лемма 4, а следовательно, и теорема 2 доказаны.

Перейдем к доказательству теоремы 1 . Для $F \in M\left(T^{2}\right), x, y, a, b \in T$ обозначим

$$
V_{a, b}(x, y):=\sum_{k=1}^{[\pi /|a|]} \sum_{j=1}^{[\pi /|b|]} \frac{\left|F\left(I_{k}, \Delta_{j}\right)\right|}{k j}+\sum_{k=1}^{[\pi /|a|]} \frac{\left|F\left(I_{k}, y\right)\right|}{k}+\sum_{j=1}^{[\pi /|b|]} \frac{\left|F\left(x, \Delta_{j}\right)\right|}{j}
$$

где $I_{k}=(x+(k-1) a, x+k a), \Delta_{j}=(y+(j-1) b, y+j b) ; F(I, \Delta)$ - смешанная разность $F$ на прямоугольнике $I \times \Delta($ см. $(5)) ; F(I, y)$ - прирашение функции $F$ на интервале $I \times y$; $F(x, \Delta)$ - приращение $F$ на интервале $x \times \Delta$. Положим

$$
V_{h}(F):=\sup _{|a| \leqslant h,|b| \leqslant h,(x, y) \in T^{2}} V_{a, b}(x, y), \quad V(F):=\sup _{h>0} V_{h}(F) .
$$

Ясно, что

$$
W_{n, m}(F) \leqslant V_{h}(F), \quad \text { если } \max \left\{\frac{\pi}{n}, \frac{\pi}{m}\right\} \leqslant h .
$$

Это означает (см. теорему 2$)$, что ряд Фурье функции $F$ равномерно сходится по Прингсхейму, если вьполнено условие

$$
\lim _{h \rightarrow 0} V_{h}(F)=0 .
$$

Величина $V(F)$ имеет много общего с гармонической вариацией функции $F$, которая определяется как супремум от правой части (25) по всевозможным системам $\left\{I_{k}\right\}$ и $\left\{\Delta_{j}\right\}$ 
непересекаюшихся интервалов из $T$ (см. [6]). В одномерном случае гармоническая вариация была введена в [7], где было доказано, что ряд Фурье непрерьвной функции ограниченной гармонической вариации сходится равномерно. Равномерная сходимость по Прингсхейму двумерных рядов Фурье непрерывной функции ограниченной гармонической вариации доказана в [6]. Теорема 2 является усилением этого результата, так как для непрерьвной функции из ограниченности гармонической вариации вытекает условие (27) (см. [6]). Отметим, что это существенное усиление, так как функция, удовлетворяющая условию (27), может не иметь ограниченной гармонической вариации. Последнее вытекает из того, что, с одной стороны, гармоническая вариация инвариантна относительно замены переменных вида (3), с другой стороны, как показывает следующая лемма 5, с помошью такой замены переменных можно достичь вьполнения условия (27).

ЛЕмма 5. Для произвольной функиии $\omega(\delta)$ вида (2) существует гомеоморфизм $\tau(t)$ отрезка $T$ такой, что для произвольной функиии $F$ вида (3) выполнены условия

$$
V(F)<\infty, \quad \lim _{h \rightarrow 0} V_{h}(F)=0 .
$$

Более того, условия (28) выполнены равномерно на множестве функиий $F$ ви$\partial a(3)$.

ДокАЗАТЕЛЬСТВо. Выберем последовательность $\left\{\delta_{n}\right\}$ так, чтобы

$$
0<4 \delta_{n+1}<\delta_{n}<1, \quad \omega\left(\delta_{n}\right) \leqslant 4^{-n} \varepsilon_{n+1}^{2}, \quad \text { где } \varepsilon_{n}=4^{-n^{2}}, \quad n=1,2, \ldots
$$

Индукцией по $n=0,1, \ldots$ построим множества

$$
T_{n}=\left\{t_{n}^{i}\right\}_{i=0}^{4^{n}}, \quad \Lambda_{n}=\left\{\lambda_{n}^{i}\right\}_{i=0}^{4^{n}} .
$$

Пусть $t_{0}^{0}=\lambda_{0}^{0}=-\pi, t_{0}^{1}=\lambda_{0}^{1}=\pi$. Считая, что $T_{n-1}$ и $\Lambda_{n-1}$ уже построены, положим

$$
\begin{aligned}
& t_{n}^{4 i}=t_{n-1}^{i} \\
& \lambda_{n}^{4 i}=\lambda_{n-1}^{i}, \\
& i=0, \ldots, 4^{n-1}, \\
& t_{n}^{4 i-1}=t_{n-1}^{i}-\varepsilon_{n}, \\
& \lambda_{n}^{4 i-1}=\frac{\lambda_{n-1}^{i-1}+\lambda_{n-1}^{i}}{2}+\delta_{n}, \quad i=1, \ldots, 4^{n-1}, \\
& t_{n}^{4 i-2}=\frac{t_{n-1}^{i-1}+t_{n-1}^{i}}{2} \\
& \lambda_{n}^{4 i-2}=\frac{\lambda_{n-1}^{i-1}+\lambda_{n-1}^{i}}{2}, \\
& i=1, \ldots, 4^{n-1}, \\
& t_{n}^{4 i-3}=\frac{t_{n-1}^{i-1}+t_{n-1}^{i}}{2}-\varepsilon_{n}, \quad \lambda_{n}^{4 i-3}=\lambda_{n-1}^{i-1}+\delta_{n}, \\
& i=1, \ldots, 4^{n-1} .
\end{aligned}
$$

Легко видеть, что множества $T_{n}$ и $\Lambda_{n}$ разбивают отрезок $T$ на интервалы

$$
d_{n}^{i}=\left(t_{n}^{i-1}, t_{n}^{i}\right), \quad \rho_{n}^{i}=\left(\lambda_{n}^{i-1}, \lambda_{n}^{i}\right), \quad i=1, \ldots, 4^{n}
$$

длины которых удовлетворяют условиям

$$
\left|d_{n}^{2 i-1}\right|=\varepsilon_{n}, \quad \frac{\varepsilon_{n-1}}{4} \leqslant\left|d_{n}^{2 i}\right| \leqslant 2^{-n}, \quad\left|\rho_{n}^{2 i-1}\right| \leqslant 2^{-n}, \quad\left|\rho_{n}^{2 i}\right|=\delta_{n}, \quad i=1, \ldots, 4^{n-1 / 2} .
$$

Положим

$$
\tau\left(t_{n}^{i}\right)=\lambda_{n}^{i}, \quad i=0, \ldots, 4^{n}, \quad n=0,1, \ldots .
$$

Из (30) следует, что функция $\tau$ определена и возрастает на объединении множеств $T_{n}$, которое всюду плотно в $T$. Так как множество значений этой функции также всюду плотно в $T$, ее можно продолжить на $T$ и получить непрерьвную функцию вида (1). Ясно, что при этом

$$
\tau\left(d_{n}^{i}\right)=\rho_{n}^{i}, \quad i=1, \ldots, 4^{n}, \quad n=0,1, \ldots
$$


ЗАмЕчАниЕ 3. Формулы (30) и (31) показывают, что гомеоморфизм $\tau$ переводит большие интервалы $d_{n}^{2 i}$ в очень маленькие, а маленькие интервалы $d_{n}^{2 i-1}$ - в сравнительно большие. Эта замена переменной делает непрерывную функцию очень близкой к кусочно-постоянной. Такой метод построения гомеоморфизма был применен Б. С. Кашиным (см. [3]) для улучшения скорости убывания коэффициентов Фурье, а также Каханом и Кацнельсоном в [2].

Допустим теперь, что $F$ - функция вида $(3)$, и докажем $(28)$. Оценим $V_{a, b}(x, y)$ для $0<a \leqslant b \leqslant h$. Выберем $n$ и $m$ так, чтобы

$$
\varepsilon_{n+1}<a \leqslant \varepsilon_{n}, \quad \varepsilon_{m+1}<b \leqslant \varepsilon_{m}, \quad m \leqslant n .
$$

Рассмотрим первую сумму в (25):

$$
A:=\sum_{k \in \Omega(a), j \in \Omega(b)} \frac{\left|F\left(I_{k}, \Delta_{j}\right)\right|}{k j}, \quad \begin{array}{ll}
\Omega(a):=\left\{1, \ldots,\left[\frac{\pi}{a}\right]\right\}, & \# \Omega(a) \leqslant \frac{\pi}{\varepsilon_{n+1}} \\
& \Omega(b):=\left\{1, \ldots,\left[\frac{\pi}{b}\right]\right\}, \quad \# \Omega(b) \leqslant \frac{\pi}{\varepsilon_{m+1}}
\end{array}
$$

(здесь и далее, \#E - количество элементов конечного множества $E$ ). Положим

$$
\begin{gathered}
\Omega_{1}(a)=\left\{k \in \Omega(a): I_{k} \subset d_{n}^{2 i} \text { или } I_{k} \subset d_{n+1}^{2 i} \text { при некотором } i\right\}, \\
\Omega_{2}(a)=\left\{k \in \Omega(a) \backslash \Omega_{1}(a): \operatorname{dist}\left(I_{k}, x\right)>\frac{\varepsilon_{n-1}}{4}\right\}, \quad \Omega_{3}(a)=\Omega(a) \backslash \Omega_{1}(a) \backslash \Omega_{2}(a) .
\end{gathered}
$$

Аналогично определяются множества $\Omega_{\nu}(b), \nu=1,2,3$ (с $m$ вместо $n$ и $b$ вместо $\left.a\right)$.

Из (30) и (31) следует, что

$$
\left|F\left(I_{k}, \Delta_{j}\right)\right| \leqslant \begin{cases}2 \omega\left(\delta_{n}\right) & \text { при } k \in \Omega_{1}(a) \\ 2 \omega\left(\delta_{m}\right) & \text { при } j \in \Omega_{1}(b), \\ 2 \omega\left(2^{-m}\right) & \text { при } k \in \Omega(a), j \in \Omega(b) .\end{cases}
$$

Легко видеть, что множество $\left\{I_{k}: k \notin \Omega_{1}(a)\right\}$ состоит из тех интервалов $I_{k}$, которые пересекаются с одним из интервалов $d_{n}^{2 i-1}$, при этом с каждым из интервалов $d_{n}^{2 i-1}$ пересекаются не более пяти интервалов, а в $\Omega_{3}(a)$ входят те интервалы, которые пересекаются с ближайшим к $x$ интервалом $d_{n}^{2 i-1}$. Поэтому

$$
\# \Omega_{2}(a) \leqslant 5 \cdot 4^{n}, \quad \# \Omega_{3}(a) \leqslant 5 .
$$

Разобьем $A$ на суммы:

$$
\begin{aligned}
A= & \sum_{k \in \Omega_{1}(a), j \in \Omega(b)}+\sum_{k \in \Omega_{2}(a), j \in \Omega(b)}+\sum_{k \in \Omega_{3}(a), j \in \Omega_{1}(b)}+\sum_{k \in \Omega_{3}(a), j \in \Omega_{2}(b)}=: A_{1}+A_{2}+A_{3}+A_{4}+A_{5} .
\end{aligned}
$$

Из (29), (32)-(34) получим

$$
\begin{aligned}
& A_{1} \leqslant 2 \omega\left(\delta_{n}\right)(\# \Omega(a))(\# \Omega(b)) \leqslant C \omega\left(\delta_{n}\right) \frac{1}{\varepsilon_{n+1}^{2}} \leqslant C 4^{-n} \\
& A_{3} \leqslant 5(\# \Omega(b)) \omega\left(\delta_{m}\right) \leqslant C \omega\left(\delta_{m}\right) \frac{1}{\varepsilon_{m+1}} \leqslant C 4^{-m}
\end{aligned}
$$


Далее, в силу (32) для $k \in \Omega_{2}(a), j \in \Omega_{2}(b)$ имеем

$$
k>\frac{\varepsilon_{n-1}}{4 \varepsilon_{n}}, \quad j>\frac{\varepsilon_{m-1}}{4 \varepsilon_{m}} .
$$

Следовательно,

$$
\begin{aligned}
& A_{2} \leqslant C \sum_{k \in \Omega_{2}(a)} \frac{4 \varepsilon_{n}}{\varepsilon_{n-1}} \omega\left(2^{-m}\right) \ln (\# \Omega(b)) \leqslant C \omega\left(2^{-m}\right) 4^{-2 n} n^{2} 4^{n}, \\
& A_{4} \leqslant 5 \omega\left(2^{-m}\right) \frac{4 \varepsilon_{m}}{\varepsilon_{m-1}}\left(\# \Omega_{2}(b)\right) \leqslant C \omega\left(2^{-m}\right) 4^{-2 m} 4^{m} .
\end{aligned}
$$

Наконец, в силу (33) и (34) выполнено $A_{5} \leqslant 25 \omega\left(2^{-m}\right)$. Суммы $A_{1}$ и $A_{3}$ в $(25)$ оцениваются аналогично. Из этих оценок находим, что

$$
V_{h}(F) \leqslant C\left(4^{-m}+\omega\left(2^{-m}\right)\right), \quad \varepsilon_{m+1}<h \leqslant \varepsilon_{m} .
$$

Так как $m \rightarrow \infty$ при $h \rightarrow 0$, отсюда вытекает (28) (равномерно по функциям $F$ вида $(3)$ ). Лемма 5 доказана.

Теорема 1 следует из лемм 4, 5 и (26). При этом условие (4) вьполняется (при достаточно большом $N$ ) для всех $F$ вида $(3)$ с $\|F\|_{\infty} \leqslant \omega(2 \pi)$. В случае произвольной $F$ достаточно рассмотреть функцию $F(x, y)-F(0,0)$. Теорема 1 доказана.

\section{СПИСОК ЦИТИРОВАННОЙ ЛИТЕРАТУРЫ}

[1] Бари Н. К. Тригонометрические ряды. М.: Физматгиз, 1961.

[2] Kahane J.-P., Katznelson Y. Series de Fourier des functions bornées // Stud. Pure Math. A la Memoire de P. Turan. Akad. Kiadó: Budapest, 1983. P. 395-410.

[3] Саакян А. А. О свойствах коэффициентов Фурье суперпозиции функций // Докл. АН СССР. 1979. T. 248. № 2. C. 302-306.

[4] Саакян А. А. О теореме Бора для кратных тригонометрических рядов // Матем. заметки. 1989. Т. 46. № 2. С. 94-103.

[5] Голубов Б.И. Двойные ряды Фурье и функции ограниченной вариации // Изв. вузов. Матем. 1972. № 12 (127). С. 55-68.

[6] Саакян А.А. О сходимости двойных рядов Фурье функций ограниченной гармонической вариации // Изв. АН АрмССР. Матем. 1986. Т. 21. №6. С. 517-529.

[7] Waterman D. On convergence of Fourier series of functions of generalized bounded variation // Studia Math. 1976. V. 44. № 1. P. 107-117.

Институт математики НАН Армении

Поступило

E-mail: sart@instmath.sci.am 10.03 .98 